BNL-NT-04/9

TAUP-2766-04

\title{
QCD in curved space-time: a conformal bag model
}

\author{
Dmitri Kharzeev \\ Physics Department, \\ Brookhaven National Laboratory, \\ Upton, NY 11973-5000, USA \\ Eugene Levin \\ HEP Department, School of Physics and Astronomy \\ Tel-Aviv University, Ramat Aviv, 69978, Israel \\ Kirill Tuchin \\ Physics Department, \\ Brookhaven National Laboratory, \\ Upton, NY 11973-5000, USA
}

(Dated: October 28, 2018)

\begin{abstract}
We construct an effective low energy Lagrangian of gluodynamics which (i) satisfies all constraints imposed by the Renormalization Group; (ii) is scale and conformally invariant in the limit of vanishing vacuum energy density; (iii) matches onto the perturbative theory at short distances. This effective theory has a dual description as classical gluodynamics on a curved conformal background. Color fields are dynamically confined, and the strong coupling freezes at distances larger than the glueball size. We also make specific predictions (in particular, on the $N_{c}$ dependence of glueball properties) which can be tested in lattice simulations of gluodynamics.
\end{abstract}

\section{INTRODUCTION}

\section{A. Instability of the perturbative vacuum}

It has been known for a long time that the perturbative QCD vacuum is not the true vacuum of the theory. One way to see this is to examine the derivation of the asymptotic freedom [1] in the effective potential method. The real part of the one-loop potential of 
gluodynamics for a constant chromomagnetic field $H$ reads [2]

$$
\operatorname{Re} V_{\text {pert }}(H)=\frac{1}{2} H^{2}+(g H)^{2} \frac{b}{32 \pi^{2}}\left(\ln \frac{g H}{\mu^{2}}-\frac{1}{2}\right),
$$

where $\mu$ is the renormalization scale, and $b=11 N_{c} / 3$. This potential has a minimum at $H=H_{0}$ : (11)

$$
g H_{0}=\mu^{2} e^{-\frac{16 \pi^{2}}{b g^{2}(\mu)}}
$$

which is natural to identify with the perturbative vacuum.

However it was soon realized that this perturbative vacuum is unstable. It is instructive to trace the origin of this instability in the effective potential method, which was pointed out in Ref. 3]. Consider the Landau levels of a particle of spin $s$ and four-momentum $p$ in a constant chromomagnetic field $H \hat{z}[4]$ :

$$
p^{\mu} p_{\mu}=2 g H(n+1 / 2)-2 s_{z} g H
$$

where $s_{z}$ is a projection of the spin on the direction of the chromomagnetic field. The effective potential (11) can be calculated as [3]

$$
V_{\text {pert }}(H)=\frac{g H}{4 \pi^{2}} \int d p_{z} \sum_{n=0}^{\infty} \sum_{s_{z}= \pm 1} \sqrt{2 g H\left(n+1 / 2-s_{z}\right)+p_{z}^{2}} .
$$

Its real part yields (11), while the imaginary part can be calculated as

$$
\operatorname{Im} V_{\text {pert }}(H)=\frac{g H}{4 \pi^{2}} \int_{-g H}^{+g H} d p_{z} \sqrt{p_{z}^{2}-g H-i 0}=-\frac{g^{2} H^{2}}{8 \pi} .
$$

Therefore, the perturbative vacuum corresponding to the minimum of the perturbative potential (2) is unstable. The instability is caused by the mode $n=0$ and $s_{z}=1$ (spin direction parallel to the field); note that $n=0$ corresponds to Landau level of the largest radius $\sim 1 / \sqrt{g H}$, i.e. to the infrared region of the theory. This means that perturbative QCD is ill-defined at large distances [5], and we may have to describe the theory in terms of other variables.

\section{B. QCD in a cavity}

The breakdown of the perturbative approach (at least, at the one-loop level) has to happen at some critical value of the chromomagnetic field $H_{c}>H_{0}=\Lambda_{Q C D}^{2} / g$. This means 
that weaker color fields cannot penetrate the physical vacuum, and the necessary condition for the applicability of the perturbative approach is that the energy density of the color field is sufficiently high:

$$
\epsilon_{H}=\frac{H^{2}}{8 \pi}>\frac{\Lambda_{Q C D}^{4}}{32 \pi^{2} \alpha_{s}}
$$

The condition (66) means that the color fields can be properly defined only at distances smaller than $R_{\text {conf }} \sim \Lambda_{Q C D}^{-1}$. For perturbative theory to make sense, it has therefore be constrained within a cavity of radius $R_{\text {conf }}$, with appropriate boundary conditions. A possible realization of this idea is the MIT bag model [6] where the colored fields are required to vanish at the surface of a sphere.

It is well known that the presence of boundary conditions leads to the emergence of Casimir vacuum energy $\epsilon_{C} \sim R_{\text {conf }}^{-4} \sim \Lambda_{Q C D}^{4}$; in MIT bag model, it is represented by a "bag constant". It is also known that a theory in flat space-time in the presence of non-trivial boundary conditions can often be conveniently described as a theory in a curved background 7]. In this paper we will argue that such a description is possible for gluodynamics. We develop an effective theory which has the following dual descriptions: (i) classical gluodynamics in a curved conformal space-time background and (ii) gluodynamics in flat space-time coupled to scalar glueballs (which in this case play the role of dilatons saturating the correlation functions of the trace of the energy-momentum tensor). The representation of the effective theory in flat space-time appears quite similar to the non-topological soliton model

of Friedberg and Lee [8] which describes quarks coupled to a scalar self-interacting field, and more generally to the approach outlined in Ref. [9]; we will return to the discussion of this topic later.

It is clear that such an approach should have its limitations. Consider, for example, the dependence on the number of colors $N_{c}$ : the energy density of the gluon field $\epsilon_{H} \sim\left(N_{c}^{2}-1\right)$, whereas the Casimir vacuum energy $\epsilon_{C} \sim \Lambda_{Q C D}^{4} \sim N_{c}^{0}$. One therefore can expect that the effect of the boundary will diminish at large $N_{c}$, and so the approach may not have a smooth $N_{c} \rightarrow \infty$ limit.

\section{Renormalization group and low energy theorems}

The basic property of the perturbative effective potential (10) is its invariance under the Renormalization Group (RG) transformations. We would like to preserve this fundamental 
property at all distances [10, 11, 12]. For this purpose, we need to encode the properties of RG in a set of low energy theorems for the correlation functions of the trace of the energy momentum tensor.

Let us sketch the derivation of these theorems, as they represent the guiding principle for the construction of our effective theory. Consider an expectation value of an operator $O$ of canonical dimension $d$; it can be written down as

$$
\langle O\rangle \sim\left[M_{0} e^{-\frac{8 \pi}{b g^{2}(\mu)}}\right]^{d} .
$$

On the other hand, the dependence of the QCD Lagrangian on the coupling is

$$
\mathcal{L}_{\mathrm{QCD}}=\left(-1 / 4 g^{2}\right) \tilde{F}^{a \mu \nu} \tilde{F}_{\mu \nu}^{a}
$$

where $\tilde{F}=g F$ is the rescaled gluon field. Following Ref. [13, 14] we can write down the expectation value of the operator $O$ in the form of the functional integral and differentiate with respect to $\left(-1 / 4 g^{2}(\mu)\right)$ to get

$$
i \int d x\left\langle T\left\{O(x), \tilde{F}^{2}(0)\right\}\right\rangle=-\frac{d}{d\left(-1 / 4 g^{2}\right)}\langle O\rangle .
$$

Combining (17) and (9) we obtain the relation [13, 14]

$$
i \lim _{q \rightarrow 0} \int d x e^{i q x}\left\langle 0\left|T\left\{O(x), \frac{\beta\left(\alpha_{s}\right)}{4 \alpha_{s}} F^{2}(0)\right\}\right| 0\right\rangle_{\text {connected }}=\langle O\rangle(-4) .
$$

This expression can be easily iterated by consequent differentiation like in (9) to obtain a set of relations between Green's functions involving an arbitrary number of operators $F^{2}$. We can rewrite those relations using expression for the scale anomaly in QCD in terms of the trace of the energy momentum tensor $\theta_{\mu}^{\mu}(d=4)$

$$
\theta_{\mu}^{\mu}=\frac{\beta(g)}{2 g} F_{\mu \nu}^{a} F^{a \mu \nu} .
$$

Substituting also $\theta_{\mu}^{\mu}$ for $O$ we obtain the following set of low energy theorems for different Green's functions involving operator $\theta_{\mu}^{\mu}(x)$ :

$$
i^{n} \int d x_{1} \ldots d x_{n}\left\langle 0\left|T\left\{\theta_{\mu_{1}}^{\mu_{1}}\left(x_{1}\right), \ldots, \theta_{\mu_{n}}^{\mu_{n}}\left(x_{n}\right), \theta_{\mu}^{\mu}(0)\right\}\right| 0\right\rangle_{\text {connected }}=\left\langle\theta_{\mu}^{\mu}(x)\right\rangle(-4)^{n} .
$$

Eqs. (10),(12) show that although the scale symmetry of the classical Yang-Mills (8) has been broken down by quantum fluctuations [15], there still remains a symmetry imposed by the invariance of the observables under the Renormalization Group. In the next section we are going to construct an effective Lagrangian which saturates (12) at long distances and matches onto the one-loop perturbative effective Lagrangian at short distances. 


\section{EFFECTIVE LAGRANGIAN}

We start with the derivation of the effective Lagrangian using the mathematical trick suggested in [11]. Consider the Yang-Mills theory on a curved conformally flat background in $d$ dimensions. The background is given by the metric

$$
\mathrm{g}_{\mu \nu}(x)=e^{h(x)} \delta_{\mu \nu},
$$

and the action by

$$
S=-\frac{1}{4 g^{2}} \int d^{d} x \sqrt{-\mathrm{g}} \mathrm{g}^{\mu \nu} \mathrm{g}^{\lambda \sigma} \tilde{F}_{\mu \lambda}^{a} \tilde{F}_{\nu \sigma}^{a} .
$$

where $\mathrm{g}=\operatorname{det} \mathrm{g}_{\mu \nu}$. Recall that the classical Yang-Mills Lagrangian in flat spacetime is scale and conformally invariant only in four dimensions. On the contrary, it can be proven 11] that the theory on the curved background given by (13),(14) is scale and conformally invariant in any number of dimensions $d$ - this means that regularization does not bring into the theory (14) any dimensionful parameters. Upon regularization the action (14) acquires an additional term in $d=4$ :

$$
\Delta S=-\frac{1}{4 g^{2}} \int d^{4} x e^{2 h}\left[-\frac{b g^{2}}{32 \pi^{2}}\left(\tilde{F}_{\mu \nu}^{a}\right)^{2}\right] .
$$

The effective one-loop action of Yang-Mills field in the external constant conformally flat gravitational field is given by the sum of (14) and (15); it is obviously scale and conformally invariant. The term (15) corresponds to the anomalous second term in the right hand side of (11). Therefore, the scale anomaly of QCD manifests itself in the theory defined by (13) and (14) through a term containing the axillary scalar field $h$ [11], without any dimensionful parameters. In a dual, and more conventional, flat space-time description the scale anomaly exhibits itself in the phenomenon of dimensional transmutation, which brings in a dimensionful parameter explicitly.

The kinetic part for the field $h(x)$ can be obtained in a manifestly scale and conformally invariant way using the Einstein-Hilbert Lagrangian for the one-loop effective Yang-Mills field

$$
S=\int d^{4} x \sqrt{-\mathrm{g}}\left(\frac{1}{8 \pi G} R-\frac{1}{4 g^{2}} \mathrm{~g}^{\mu \nu} \mathrm{g}^{\lambda \sigma} \tilde{F}_{\mu \lambda}^{a} \tilde{F}_{\nu \sigma}^{a}-e^{2 h} \theta_{\mu}^{\mu}\right),
$$

where $R$ is the Ricci scalar and $G$ is some dimensionful constant; we substituted (111) into the square brackets of (15). We can now use a well-known expression for the Riemann tensor 
$R_{\mu \nu}$ [25] to write down the dynamical terms for the field $h(x)$ which obey the scale and conformal symmetry. Using (13) we get

$$
R \sqrt{-\mathrm{g}} \equiv R_{\mu}^{\mu} \sqrt{-\mathrm{g}}=e^{h} \frac{3}{2}\left(\partial_{\mu} h\right)^{2} .
$$

Note that by writing (17) we explicitly neglected terms of higher order in derivatives and constrained ourselves to the Einstein's gravity. This correspond to an expansion in powers of a slowly varying background field.

The vacuum expectation value of the energy-momentum tensor reads

$$
\left\langle\theta_{\mu}^{\mu}\right\rangle=-4\left|\epsilon_{\mathrm{v}}\right|
$$

The perturbative contribution to (18) is given by (11). Since the perturbative vacuum (2) is not stable, it is natural to assume that the dominant contribution to the energy density of the physical vacuum comes from non-perturbative modes. It is therefore convenient to separate the perturbative contribution to the $\theta_{\mu}^{\mu}$ in the following way:

$$
\theta_{\mu}^{\mu}=\theta_{\mu}^{\mu}(\text { pert. })-4\left|\epsilon_{\mathrm{v}}\right|
$$

(we will argue below (see (25) ) that the physical vacuum is indeed independent of the value of the external chromomagnetic field.) Combining (16), (17) and (19) we arrive at the expression for the effective one-loop action in the conformally flat gravitational field

$$
S=\int d^{4} x\left(\frac{4\left|\epsilon_{\mathrm{v}}\right|}{m^{2}} e^{h}\left(\partial_{\mu} h\right)^{2}-\frac{1}{4}\left(F_{\mu \nu}^{a}\right)^{2}+\left|\epsilon_{\mathrm{v}}\right| e^{2 h}-\frac{1}{4} e^{2 h}\left[-\frac{b g^{2}}{32 \pi^{2}}\left(F_{\mu \nu}^{a}\right)^{2}\right]\right),
$$

where the new dimensionful constant $m$ was introduced instead of $G$ 16].

At this point it is important to note that one can easily read the running coupling constant off (14) and (15). It can be seen that $-e^{2 h}$ plays a role of the familiar perturbative logarithm $2 \ln \left(q^{2} / \mu^{2}\right)$. Hence our effective theory is applicable when $q^{2}<\mu^{2}$. In the infrared region the perturbative expressions break down. However it is possible to remove the explicit dependence on the strong coupling from the effective action by performing the following redefinition in (20)

$$
h \rightarrow h-2 \ln \varsigma, \quad\left|\epsilon_{\mathrm{v}}\right| \rightarrow \varsigma^{4}\left|\epsilon_{\mathrm{v}}\right|, \quad m^{2} \rightarrow \varsigma^{2} m^{2},
$$

where $\varsigma^{4}=g^{2} b / 32 \pi^{2}$. Eq. (21) is just a change of mass unit. 
Finally, we have to perform Legendre transformation of the action (201) to get the minimum of the effective potential at the minimum of the field $\chi$ which is canonically conjugated to the field $h$ [1]]. The result reads [16]

$$
\mathcal{L}=\frac{\left|\epsilon_{\mathrm{v}}\right|}{m^{2}} \frac{1}{2} e^{\chi / 2}\left(\partial_{\mu} \chi\right)^{2}+\left|\epsilon_{\mathrm{v}}\right| e^{\chi}(1-\chi)-\frac{1}{4}\left(F_{\mu \nu}^{a}\right)^{2}+e^{\chi}(1-\chi) \frac{1}{4}\left(F_{\mu \nu}^{a}\right)^{2},
$$

where we included the factor $\sqrt{-\mathrm{g}}$ in the definition of $\mathcal{L}$. The Lagrangian (22) defines our effective theory. At large distances (22) reduces to the low energy effective Lagrangian of Refs. [11, 12]. Indeed, the Yang-Mills action is scale invariant in the external field $h$, which implies that $F_{\mu \nu}^{a} \sim q^{2} \rightarrow 0$ at long distances. We will investigate the region of applicability of the effective Lagrangian (22) in the following sections.

The mathematical trick of putting the theory in curved space-time background which we used in derivation of (22) gives a simple way to keep track of all symmetries of the effective Lagrangian. However, we think it is also instructive to check how the effective perturbative potential (11) emerges from the Lagrangian (22). The energy density $\theta^{00}$ corresponding to (22) is given by

$$
\begin{aligned}
\theta^{00}(x)= & \frac{\left|\epsilon_{\mathrm{v}}\right|}{2 m^{2}}\left[\left(\partial_{0} \chi\right)^{2}+\left(\partial_{i} \chi\right)^{2}\right] e^{\chi / 2}-\mathrm{g}^{00}\left|\epsilon_{\mathrm{v}}\right| e^{\chi}(1-\chi) \\
& +\left(-F^{a 0 \lambda} F_{\lambda}^{a 0}+\frac{1}{4} \mathrm{~g}^{00}\left(F_{\lambda \sigma}^{a}\right)^{2}\right)\left(1-e^{\chi}(1-\chi)\right),
\end{aligned}
$$

where $i=1,2,3$. Therefore the effective potential $W$ in the constant chromomagnetic field $H$ is

$$
W=\int d^{3} x\left\{\frac{1}{2} H^{2}-e^{\chi}(1-\chi)\left(\frac{1}{2} H^{2}+\left|\epsilon_{\mathrm{v}}\right|\right)\right\} .
$$

In strong chromomagnetic field $H^{2} \gg\left|\epsilon_{\mathrm{v}}\right|$ the energy density $W$ reduces to the effective potential (II). In this case $\chi$ is not an independent degree of freedom, but rather a function of $H$. We calculate the corresponding momentum scale in the next section. The minimum of the functional $W(H, \chi)$ is found from the following equations

$$
1-e^{\chi}(1-\chi)=0, \quad \chi e^{\chi}\left(\frac{1}{2} H^{2}+\left|\epsilon_{\mathrm{v}}\right|\right)=0 .
$$

Evidently, the minimum is at $\chi=0$ and the value of the $W$ at the minimum is $-\left|\epsilon_{\mathrm{v}}\right|$ independently of the value of the chromomagnetic field $H$. We conclude that the physical vacuum of the gluodynamics is described by one scalar field even in the presence of the applied chromomagnetic field. This justifies our assumption (19). 
It is seen from (24) that an increase of the color field $H$ leads to the increase of the energy density of the system. Since $H \sim g / r^{2}$ (where $r$ is the size of the system) the energy density decreases with $r$. At the same time the volume which the system occupies increases as $r^{3}$. Therefore, we expect that there exists a static configuration with a finite size $r_{0}$ such that the total energy of the system in minimal. This is analogous to the mechanism of bag formation in the Friedberg-Lee model [8]. However, the minimum of the effective potential in our model is independent of $H$ and located at $\chi=0$, while in the Friedberg-Lee model it depends on the density of the color sources.

Note that we can read the one-loop behavior of the strong coupling right off the expression (11) for the effective potential. Indeed, the susceptibility of the vacuum in the strong external chromomagnetic field is (do not confuse $\mu(H)$ with the renormalization scale $\mu$ in (11)

$$
\mu(H)=1-\frac{\beta(g)}{g}\left(\ln \frac{g H}{\mu^{2}}-\frac{1}{2}\right)
$$

Recall that the beta function can be interpreted as a response of the system to the change of the external field. Namely, (26) implies

$$
\beta=-g \frac{\partial \mu(H)}{\partial \ln H}
$$

From (22) it follows that $\mu(H)$ is independent of $H$ at long distances, therefore $\beta=0$. The strong coupling does not run if the effective theory is considered at the tree level. We will see in the next section that quantum corrections do not alter that conclusion.

It remains to check that the vacuum at $\chi=0$ is stable. Let us recall that (11) is the real part of the perturbative effective potential. However the perturbative potential has also the imaginary part, as discussed above, which is due to the instability of the Landau level with $n=0$ and $s_{z}=1$, i.e. spin direction is parallel to the field.

Let us now examine the properties of the Landau levels in our effective theory near the $\chi=0$. The equation of motion of the dilaton field is

$$
\frac{\left|\epsilon_{\mathrm{v}}\right|}{m^{2}} \partial_{\mu}\left(e^{\chi / 2} \partial_{\mu} \chi\right)-\frac{\left|\epsilon_{\mathrm{v}}\right|}{4 m^{2}} e^{\chi / 2}\left(\partial_{\mu} \chi\right)^{2}+\chi e^{\chi}\left|\epsilon_{\mathrm{v}}\right|+\chi e^{\chi} \frac{1}{4} F_{\mu \nu}^{a} F^{a \mu \nu}=0 .
$$

Expanding near the minimum we arrive at

$$
\partial_{\mu}^{2} \chi+m^{2}\left(1+\frac{H^{2}}{2\left|\epsilon_{\mathrm{v}}\right|}\right) \chi=0 .
$$


The corresponding Landau levels are

$$
p^{\mu} p_{\mu}=m^{2}\left(1+\frac{H^{2}}{2\left|\epsilon_{\mathrm{v}}\right|}\right) .
$$

It is seen that $p_{\mu} p^{\mu} \geq 0$ for any $H$ so that the instability does not develop in the effective theory we are discussing in this paper.

Next, consider the trace of energy-momentum tensor which can be calculated directly from (22) using

$$
\theta_{\mu}^{\mu}=\delta^{\mu \nu}\left(2 \frac{\partial \mathcal{L}}{\partial \delta^{\mu \nu}}-\delta^{\mu \nu} \mathcal{L}\right)+\frac{8\left|\epsilon_{\mathrm{v}}\right|}{m^{2}} \partial_{\mu}^{2} e^{\chi / 2},
$$

where the last term in the right hand side is the total derivative. Using equation of motion of the dilaton field (28) one arrives at

$$
\theta_{\mu}^{\mu}=-4\left|\epsilon_{\mathrm{v}}\right| e^{\chi}-\chi e^{\chi} F_{\mu \nu}^{a} F^{a \mu \nu}
$$

By virtue of (28) one can clearly see that in the limit $\left|\epsilon_{\mathrm{v}}\right| \rightarrow 0$ the trace (32) vanishes and the classical symmetries of the Yang-Mills Lagrangian are restored. One might be worried that in the expansion of (32) in powers of $\chi$ the term $\chi F_{\mu \nu}^{a} F^{a \mu \nu}$ appears while it is absent in the Lagrangian (22). However it is easy to see that this term is canceled out by the pure dilaton contribution. Indeed, expanding the equation of motion (28) up to the quadratic terms in $\chi$ one finds

$$
\frac{1}{4} F_{\mu \nu}^{a} F^{a \mu \nu} \chi=-\left|\epsilon_{\mathrm{v}}\right| \chi+\text { full derivative }+\mathcal{O}\left(\chi^{2}\right)
$$

It is important to check that $\theta_{\mu}^{\mu}$ satisfies the low energy theorems (12). We have noted just after (22) that $F_{\mu \nu}^{a} \sim q^{2}$. Hence it does not contribute to the $\theta_{\mu}^{\mu}$ at $q \rightarrow 0$. The only remaining term in (32) is the first one; it saturates the low energy theorems as was explicitly proved in [11]. Thus, we have at our disposal the effective Lagrangian which (i) satisfies the constraints imposed by the Renormalization Group, (32); (ii) the classical minimum $\chi=0$ of its effective potential (24) is the true stable vacuum of QCD; (iii) its scale and conformal symmetry is restored if $\left|\epsilon_{\mathrm{v}}\right|=0$.

\section{QUANTUM FLUCTUATIONS AROUND THE PHYSICAL VACUUM}

Effective theory (22) is non-renormalizable. Let $M_{0}$ be its UV cutoff (in the effective potential method, this is the scale which corresponds to the lowest Landau level). Quantum 
fluctuations can develop only if there is enough kinematical space which is the case if $m \ll M$. Let us define the perturbative expansion parameter $\lambda$ as

$$
\lambda=\frac{m}{M_{0}} .
$$

We will see later in this section that perturbative series in powers of $\lambda$ is equivalent to the expansion of the Lagrangian (22) in powers of $\chi$, and $\lambda$ indeed is the small expansion parameter in our effective theory. For the rest of this section we assume that $\lambda$ is small and prove this assumption in section IV]

\section{A. Normalization of the energy-momentum tensor}

Let us first find the scale $M_{0}$ at which our effective description breaks down; we will work in the leading order in $\lambda$. The vacuum expectation value of the trace of energy-momentum tensor (32) is the physical observable and does not depend on a particular choice of degrees of freedom in the Lagrangian; its value is given by (18). By virtue of (32) it is equivalent to the requirement that

$$
4\left|\epsilon_{\mathrm{v}}\right|\left\langle 1-e^{\chi}\right\rangle=\left\langle\chi e^{\chi} F_{\mu \nu}^{a} F^{a \mu \nu}\right\rangle .
$$

In the vacuum $\chi=0$ (18) is obviously satisfied. Quantum fluctuations in general violate this requirement. However, since the effective Lagrangian (22) is formally divergent at short distances we have to impose an ultra-violet cutoff $M_{0}$. We will choose such a cutoff that (18) is satisfied.

Expanding (32) to the order $\mathcal{O}\left(\chi^{0}\right)$ we obtain a trivial result

$$
\theta_{\mu}^{\mu}=-4\left|\epsilon_{\mathrm{v}}\right|+\mathcal{O}(\chi)
$$

At the order $\mathcal{O}(\chi)$ Eq. (35) is satisfied due to (33). At the next order $\mathcal{O}\left(\chi^{2}\right)$ (35) can be satisfied only for a particular choice of the cutoff $M_{0}$. Note that by the low energy theorems (12) (with $n=1$ ) long distance contributions to expectation value of the operator $\theta_{\mu}^{\mu}(x)$ can be expressed through the two point correlator $\Xi\left(q^{2}\right)$ defined as

$$
\Xi\left(q^{2}\right)=i \int d^{4} x e^{i q x}\left\langle 0\left|T \theta_{\mu}^{\mu}(x) \theta_{\mu}^{\mu}(0)\right| 0\right\rangle=\int d \sigma^{2} \frac{\rho\left(\sigma^{2}\right)}{\sigma^{2}-q^{2}-i 0},
$$

where we have introduced the spectral density $\rho\left(q^{2}\right)$. We find it more convenient to work with this correlator. The first reason is that the spectral density can be expressed in terms of physical states. The other one is that we know $\rho\left(q^{2}\right)$ for the perturbative theory. 


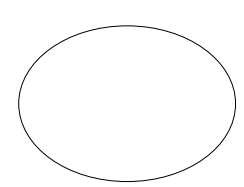

(a)

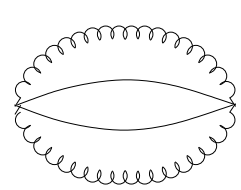

(b)

FIG. 1: (a) Pure dilaton contribution to the trace of energy-momentum tensor; (b) mixed dilatongluon contribution to the two-point correlator of the trace of energy-momentum tensor.

To rewrite condition (35) in terms of two-point correlator we apply to it the low energy theorem (12)

$$
\Xi(0)=-4\left\langle\theta_{\mu}^{\mu}\right\rangle
$$

Thus, our requirement that (35) holds at the leading non-trivial order in $\lambda$ can be written as (see (32))

$$
\Xi_{\text {dil }}(0)+\Xi_{\text {mix }}(0)=0
$$

where we separated the pure dilaton and mixed dilaton-gluon contributions.

The pure dilaton contribution can be read from (32):

$$
\left\langle\theta_{\mu}^{\mu}\right\rangle_{\text {dil }}=-4\left|\epsilon_{\mathrm{v}}\right| \frac{1}{2}\left\langle\chi^{2}\right\rangle
$$

which implies that (see Fig. 1(a))

$$
\Xi_{\text {dil }}(0)=8\left|\epsilon_{\mathrm{v}}\right|\left\langle\chi^{2}\right\rangle=8\left|\epsilon_{\mathrm{v}}\right| \frac{m^{2}}{\left|\epsilon_{\mathrm{v}}\right|} \frac{1}{2} \int \frac{d^{4} k}{(2 \pi)^{4}} \frac{i}{k^{2}}=\frac{m^{2} M_{0}^{2}}{2 \pi^{2}} .
$$

Let us turn to the mixed gluon-dilaton contributions. The corresponding diagram is shown in Fig. 1(b). Its imaginary part is

$$
\begin{aligned}
\rho_{\text {mix }}\left(\sigma^{2}\right)= & \left(\frac{m^{2}}{\left|\epsilon_{\mathrm{v}}\right|}\right)^{2} \frac{N_{c}^{2}-1}{4} \int \frac{d^{4} q_{1}}{(2 \pi)^{4}} \int \frac{d^{4} q_{2}}{(2 \pi)^{4}}\left(q_{1}^{\mu} q_{2}^{\nu}-\left(q_{1} \cdot q_{2}\right) g^{\mu \nu}\right)^{2}(2 \pi)^{2} \delta\left(q_{1}^{2}\right) \delta\left(q_{2}^{2}\right) \\
& \times \int \frac{d^{4} k_{1}}{(2 \pi)^{4}} \int \frac{d^{4} k_{2}}{(2 \pi)^{4}}(2 \pi)^{2} \delta\left(k_{1}^{2}\right) \delta\left(k_{2}^{2}\right)(2 \pi)^{4} \delta\left(k_{1}+k_{2}+q_{1}+q_{2}\right) \\
= & \frac{\sigma^{8}}{140 \cdot 48(2 \pi)^{5}}\left(N_{c}^{2}-1\right)\left(\frac{m^{2}}{\left|\epsilon_{\mathrm{v}}\right|}\right)^{2}
\end{aligned}
$$

where we neglected the mass of the dilaton $m$ with respect to the cutoff $M_{0} . \Xi\left(q^{2}\right)$ can be calculated using dispersion relation with subtractions

$$
\Xi\left(q^{2}\right)_{\text {mix }}=\int_{0}^{\infty} \frac{\rho_{\text {mix }}\left(\sigma^{2}\right) d \sigma^{2}}{\sigma^{2}-q^{2}-i 0}-q^{2} \int_{0}^{\infty} \frac{\rho_{\text {mix }}\left(\sigma^{2}\right) d \sigma^{2}}{\sigma^{4}}
$$




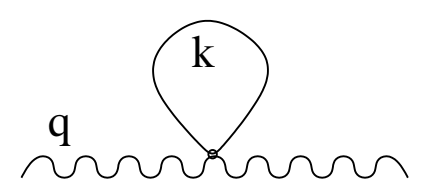

FIG. 2: The leading order quantum correction to the gluon propagator at long distances.

$$
\begin{aligned}
& -q^{4} \int_{0}^{\infty} \frac{\rho_{\text {mix }}\left(\sigma^{2}\right) d \sigma^{2}}{\sigma^{6}}-\ldots-q^{12} \int_{0}^{\infty} \frac{\rho_{\text {mix }}\left(\sigma^{2}\right) d \sigma^{2}}{\sigma^{14}} \\
= & q^{10} \int_{0}^{\infty} \frac{\rho_{\text {mix }}\left(\sigma^{2}\right) d \sigma^{2}}{\sigma^{10}\left(\sigma^{2}-q^{2}-i 0\right)}-\int_{0}^{\infty} \frac{\rho_{\text {mix }}\left(\sigma^{2}\right) d \sigma^{2}}{\sigma^{2}} .
\end{aligned}
$$

The dispersion integral in the last line of (43) is proportional to $q^{10} \ln \left(-M_{0}^{2}+q^{2}\right)$. Consequently,

$$
\Xi_{\text {mix }}(0)=-\int_{0}^{\infty} \frac{\rho_{\text {mix }}\left(\sigma^{2}\right) d \sigma^{2}}{\sigma^{2}}=-\frac{1}{4} \rho_{\text {mix }}\left(M_{0}^{2}\right) .
$$

Formally, (44) gives the value of the non-vanishing subtraction constant in the dispersion relation.

Substituting (41) and (44) into vacuum stability condition (39) results in the equation determining the ultra-violet cutoff $M_{0}$ of the effective theory [16]

$$
M_{0}^{2}=16 \pi 105^{1 / 3}\left(N_{c}^{2}-1\right)^{-1 / 3}\left(\frac{\left|\epsilon_{\mathrm{v}}\right|}{m}\right)^{2 / 3} .
$$

\section{B. Gluon polarization tensor}

We have argued that the vacuum expectation of the gluon condensate (18) is unchanged provided we had chosen the value of the cut-off according to (45). In that case the quantum corrections does not change the vacuum energy density which is completely saturated by classical solution. Now we would like to calculate quantum corrections to the strong coupling. To the leading order in $\lambda$ we have the tadpole diagram in Fig. 2] Introduce the scalar function $\Pi\left(q^{2}\right)$ as follows

$$
\Pi_{\mu \nu}(q)=\left(q^{\mu} q^{\nu}-q^{2} g^{\mu \nu}\right) \Pi(q) .
$$

The tadpole diagram is given by

$$
i \Pi_{\mu \nu}^{\mathrm{tadpole}}(q)=\frac{1}{2} \frac{m^{2}}{\left|\epsilon_{\mathrm{v}}\right|} \int_{m}^{M_{0}} \frac{d^{4} k}{(2 \pi)^{4}} \frac{i}{k^{2}-m^{2}+i 0} i(-1)\left(q^{\mu} q^{\nu}-q^{2} g^{\mu \nu}\right) .
$$


It can be calculated by performing Wick rotation and consequent integration over a four dimensional sphere of radius $M_{0}$. We neglect then the dilaton mass which gives contribution of higher order in $\lambda$. The result of calculation of the diagram in Fig. 2 is

$$
\Pi\left(q^{2}\right)=\frac{M_{0}^{4}}{64 \pi^{2}\left|\epsilon_{\mathrm{v}}\right|}, \quad q^{2} \leq M_{0}^{2} .
$$

The quantum correction is constant. This means that the strong coupling freezes at long distances.

The tadpole diagram Fig. 2 is leading order in $\lambda$ correction. However the higher order corrections deserve a special remark since they could be in principle logarithmically divergent at $q^{2}=4 m^{2}$ in which case those diagrams would dominate the polarization tensor at

long distances. In the Appendix B we argue that all such logarithms appear in the product $\left(q^{2}-4 m^{2}\right)^{n} \ln \left(q^{2} / 4 m^{2}\right)$, where $n>0$ and thus vanish at the end-point of the dilaton spectrum. Also we check that the sub-leading diagrams are numerically small. Therefore, the conclusion of the previous section that the strong coupling freezes at long distances holds if such quantum corrections are included.

Eq. (48) is the leading order contribution of vacuum fluctuations to the gluon polarization tensor. We can systematically develop the perturbation theory in $\lambda$. The qualitative picture of the renormalization group flow can be obtained by simple dimensional analysis (see e.g. 24]). Since the typical scale for mass is the cutoff $M_{0}$, the coefficients in front of the four terms in the rhs of (22) have the following behavior at different momentum scales $p:\left(M_{0} / p\right)^{2}$, $\left(M_{0} / p\right)^{4},\left(M_{0} / p\right)^{0}$ and $\left(M_{0} / p\right)^{2}$ respectively. Thus, the only relevant term at low momenta is the second one, which is purely dilatonic term. This is a manifestation of the fact that the dynamics of the vacuum fields decouples from the colored sources.

\section{MATCHING ONTO THE PERTURBATION THEORY}

One can express the strong coupling at the cutoff $M_{0}$ as function of the parameters of the low energy Lagrangian. This can be achieved by matching the spectral density of the effective theory (42) with the spectral density of perturbation theory at $M_{0}^{2}$. In perturbative gluodynamics the anomalous trace of energy-momentum tensor is given by (111). Then the calculation of the spectral density of the correlator (37) is straightforward 17]

$$
\rho_{\text {pert }}\left(q^{2}\right)=\left(\frac{b \alpha_{s}}{8 \pi}\right)^{2} 4^{2} \frac{N_{c}^{2}-1}{2} \int \frac{d^{4} q_{1}}{(2 \pi)^{4}} \int \frac{d^{4} q_{2}}{(2 \pi)^{4}}\left(q_{1}^{\mu} q_{2}^{\nu}-\left(q_{1} \cdot q_{2}\right) g^{\mu \nu}\right)^{2}
$$




$$
\begin{aligned}
& \times(2 \pi)^{2} \delta\left(q_{1}^{2}\right) \delta\left(q_{2}^{2}\right)(2 \pi)^{4} \delta\left(q+q_{1}+q_{2}\right) \\
= & \left(\frac{b \alpha_{s}}{8 \pi}\right)^{2} \frac{\left(N_{c}^{2}-1\right)}{2 \pi} q^{4} .
\end{aligned}
$$

Since the spectral density is just the imaginary part of the correlator, it is clear that only mixed diagrams of (44) contribute to the matching in the leading in $\lambda$ order (indeed, $\rho_{\text {dil }} \sim$ $\left.m^{4}\right)$

$$
\rho_{\text {mix }}\left(M_{0}^{2}\right)=\rho_{\text {pert }}\left(M_{0}^{2}\right)
$$

Using Eq. (45) we obtain

$$
\alpha_{s}\left(M_{0}^{2}\right)=\frac{16 \sqrt{\pi} \lambda}{b \sqrt{N_{c}^{2}-1}}, \quad-q^{2}=Q^{2} \leq M_{0}^{2},
$$

This equation shows that the small parameter of perturbation theory $\alpha_{s}$ is matched onto the small parameter of our effective theory, $\lambda$.

At $Q^{2}>M_{0}^{2}$ the strong coupling runs as

$$
\alpha_{s}\left(Q^{2}\right)=\frac{\alpha_{s}\left(M_{0}^{2}\right)}{\left(1+\frac{b \alpha_{s}}{4 \pi} \ln \frac{Q^{2}}{M_{0}^{2}}\right)}=\frac{4 \pi}{b \ln \frac{Q^{2}}{\Lambda_{\mathrm{QCD}}^{2}}}, \quad Q^{2}>M_{0}^{2},
$$

where we introduced the familiar phenomenological constant constant $\Lambda_{\mathrm{QCD}}$ as

$$
\Lambda_{\mathrm{QCD}}^{2}=M_{0}^{2} e^{-\frac{4 \pi}{b \alpha_{s}\left(M_{0}^{2}\right)}}
$$

\section{A. Numerical estimations}

QCD sum rules analysis performed in [13, 14] make it possible to estimate the nonperturbative scale inherent to the vacuum of gluodynamics, which appears quite hard: $M_{0}^{2}=$ $20 \mathrm{GeV}^{2}$. Lattice calculations show 20] that the lightest resonance in pure gluodynamics is the scalar glueball with mass $m \simeq 1.6 \mathrm{GeV}$. It is natural to identify this glueball with dilaton; it is interesting that this state appears to have a size much smaller than the sizes of glueballs with other quantum numbers [20]. In the approach followed in this paper this is a consequence of a large value of the cutoff scale $M_{0}$. From the vacuum stability condition (45) we find $\left|\epsilon_{\mathrm{v}}\right| \simeq(0.58 \mathrm{GeV})^{4}$. By definition $\lambda=m / M_{0} \simeq 0.36$. Eq. (151) then implies that $\alpha_{s}\left(M_{0}^{2}\right) \simeq 0.33$. The value of the $\Lambda_{\mathrm{QCD}}$ follows from (53): $\Lambda_{\mathrm{QCD}} \simeq 0.79 \mathrm{GeV}$.

In the world with light quarks the scalar glueball mixes with scalar $\bar{q} q$ meson [15]. The lightest scalar resonance is the $\sigma$-resonance which is a strong mixture of the glueball and 
the $q \bar{q}$ meson [18]. In this case the dilaton mass can be estimated as the mass of the $\sigma$ 16]: $m \simeq 0.6 \mathrm{GeV}$. QCD sum rules give an estimate of the QCD vacuum energy density: $\left|\epsilon_{\mathrm{v}}\right|=(0.24 \mathrm{GeV})^{4}$. From (45) we have $M_{0} \simeq 1.9 \mathrm{GeV}$. Other estimates can be done exactly as in the previous paragraph yielding $\lambda=0.31, \alpha_{s}\left(M_{0}^{2}\right) \simeq 0.35$ and $\Lambda_{\mathrm{QCD}}=0.26 \mathrm{GeV}$. To verify how good is this value from the phenomenological point of view we use (52) and find that at the Z-boson mass scale $\alpha_{s}\left(m_{Z}\right) \simeq 0.12$. This is in a reasonable agreement with the data - see discussion in Ref. [21].

\section{B. Dependence on $N_{c}$}

Let us now discuss the dependence of our effective theory on the number of colors $N_{c}$. Eq. (53) can be considered as an equation for the cutoff of the effective theory $M_{0}$ as a function of the number of colors $N_{c}$. Let us find $M_{0}\left(N_{c}\right)$. It is convenient to introduce the dimensionless parameter $a$ and have $\lambda$ rescaled as follows

$$
\bar{\lambda}=\frac{\lambda \Lambda_{\mathrm{QCD}}}{m}, \quad a=\sqrt{N_{c}^{2}-1} \frac{\Lambda_{\mathrm{QCD}} \sqrt{\pi}}{8 m} .
$$

Then using (134) and (54) Eq. (153) takes form

$$
\bar{\lambda}=\exp \left\{-\frac{a}{\bar{\lambda}}\right\}
$$

Its solution is shown in Fig. 3(a). We observe that the solution has two branches: one starting at the origin $(0,0)$ and the second one starting at the point $(0,1)$. Both branches terminate at the critical point $\left(a_{\mathrm{cr}}, \bar{\lambda}_{\mathrm{cr}}\right)=\left(e^{-1}, e^{-1}\right)$. To pick up the physical branch we note that by (54) $a=0$ when $N_{c}=1$. Thus, by (51) $\bar{\lambda}=0$ at this point. Therefore the physical branch is the lower one in Fig. 3)(a). In Fig. 3(b) we represent it as a plot of the cutoff $M_{0}$ versus the number of colors $N_{c}$. The value of $\Lambda_{\mathrm{QCD}}=0.8$ chosen for this figure is such that $\alpha_{s}\left(M_{Z}^{2}\right)=0.12$ at $b=11$. The critical value of the parameter $a$ corresponds to the critical value of $N_{c}$. Using (154) we find

$$
N_{c}^{2} \leq N_{c}^{\mathrm{cr} 2}=\left(\frac{8 m}{\sqrt{\pi} \Lambda_{\mathrm{QCD}}} e^{-1}\right)^{2}+1,
$$

(of course, the integer part of the right-hand-side must be taken) which yields $N_{c}^{c r}=3$. When $N_{c}>N_{c}^{c r}$ our effective theory ceases to be valid. Indeed, $M_{0}$ rapidly decreases with 


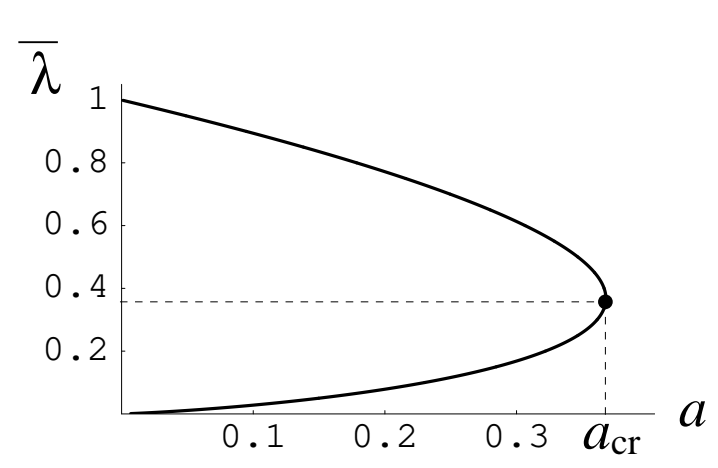

(a)

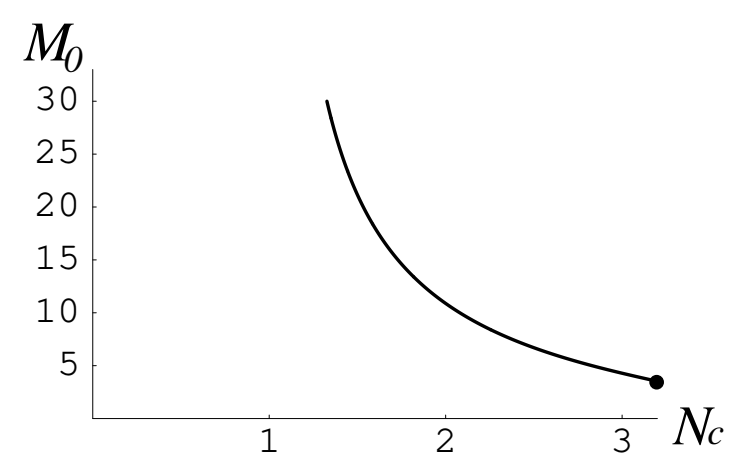

(b)

FIG. 3: Numerical solution to [55). (a) Rescaled coupling $\bar{\lambda}$ vs $a$; the critical point is $\left(e^{-1}, e^{-1}\right)$. (b) Dependence of $M_{0}$ on the number of colors in gluodynamics $\left(\Lambda_{\mathrm{QCD}}=0.8 \mathrm{GeV}\right)$; only the physical branch of the solution is shown. With a good accuracy $M_{0} \propto 1 / N_{c}^{2}$.

$N_{c}$ (approximately as $1 / N_{c}^{2}$ ) approaching the dilaton mass $m$. The values of the strong coupling $\alpha_{s}\left(M_{0}^{\mathrm{cr}}\right)$ and the cutoff $M_{0}^{\mathrm{cr}}$ at the critical point are

$$
\alpha_{s}\left(M_{0}^{\mathrm{cr}}\right)=\frac{2 \pi}{b^{\mathrm{cr}}}
$$

and

$$
M_{0}^{\mathrm{cr}}=\frac{8 m}{\sqrt{\pi\left(N_{c}^{\mathrm{cr} 2}-1\right)}} .
$$

We see that the effective theory breaks down at large $N_{c}$, with the critical value $N_{c}^{\text {cr }}$ (see (56)). This can be readily interpreted if we recall that the perturbative vacuum energy density grows as $\left|\epsilon_{\mathrm{v}}\right|^{\text {pert }} \sim N_{c}^{2}$ at large $N_{c}$, whereas $\left|\epsilon_{\mathrm{v}}\right|$ of the effective theory does not (see Fig. 3(b) and (45)). Thus, the effective theory breaks down at such large values of $N_{c}$ that the perturbative vacuum energy density cannot be matched onto the effective one. In the region $N_{c} \leq N_{c}^{c r}$ where we can use the effective theory, the $N_{c}$ dependence of the value of the freezing strong coupling is given by $\alpha_{s}\left(M_{0}^{2}\right) b \sim \lambda / N_{c} \sim N_{c}$ as can be seen from (51) and Fig. 3(b).

Since $M_{0}$ decreases as $N_{c}$ increases the matching region is driven into the infrared where the perturbative expansion can no longer be trusted. Indeed, the dilaton spectral density vanishes at $q^{2}<4 m^{2}$. On the contrary, the perturbative gluon spectral density is finite at arbitrary small but finite $q^{2}$ (see (49)). Although the dilaton effective theory takes into account the non-perturbative effects associated with the scale anomaly, it is not clear how 
those effects are related to the color potential at long distances. The interplay between the dilaton low energy effective theory and the gluodynamics at large $N_{c}$ certainly deserves special study.

\section{CONCLUSIONS}

In this paper we constructed an effective low energy Lagrangian (22) of gluodynamics which (i) satisfies the constraints imposed by the Renormalization Group; (ii) its vacuum is stable; (iii) it is scale and conformally invariant in the limit of vanishing vacuum energy density $\left|\epsilon_{\mathrm{v}}\right|$; (iv) it matches onto the perturbative theory at short distances. Using this Lagrangian we developed the perturbation theory of quantum fluctuations around the physical vacuum. Since the effective theory (22) is divergent when considered on a quantum level we must introduce an ultraviolet cutoff $M_{0}$. To calculate it we noted that classical configuration of the dilaton field saturates the vacuum energy $\left|\epsilon_{\mathrm{v}}\right|$. Therefore the value of $M_{0}$ is dictated by the requirement of vacuum stability - quantum fluctuations must not contribute to the vacuum energy density. This happens to be true only for a certain choice of $M_{0}$ given by (45). In the kinematic region $q^{2} \leq M_{0}^{2}$ we developed a perturbation theory in a small parameter $\lambda=m / M_{0}$ and used it to calculate the leading (48) and next-to-leading (B7) order radiative corrections to the gluon propagator. We observed that the leading radiative correction to the gluon propagator is constant. We conclude that the strong coupling $\alpha_{s}$ freezes at distances larger than the inverse cutoff $1 / M_{0}$; this behavior is consistent with the analysis of Refs. [21], 19] .

By matching the spectral densities of the perturbation theory and of the effective one we determined the value of the strong coupling at the scale $M_{0}$ in terms of the vacuum energy density $\left|\epsilon_{\mathrm{v}}\right|$ and the glueball mass $m$, (151). Using QCD sum rules to estimate $M_{0}^{2} \simeq 20 \mathrm{GeV}^{2}$ we calculate the $\Lambda_{\mathrm{QCD}}$ and then $\alpha_{s}\left(m_{Z}\right)$; we found a reasonable agreement with experimental data. We consider this as an additional evidence that the typical scale of vacuum fluctuations of QCD is hard [14, 17, 22].

We discussed the $N_{c}$ dependence of the theory. As $N_{c}$ increases $M_{0}$ decreases as $\sim 1 / N_{c}^{2}$, so that at some $N_{c}^{\text {cr }}$ we have $M_{0} \leq m$ and the quantum fluctuations of the dilaton field are no longer possible. The matching on the perturbation theory (50) and (151) breaks down. Numerically, $N_{c}^{c r}$ is found to be just above 3, so the effective theory (22) is applicable to the 
study the infrared behavior of $S U(3)$ gluodynamics.

One of our main results - the freezing of the strong coupling at long distances - has an elegant geometric interpretation. Recall that we derived the effective Lagrangian (22) by formally coupling Yang-Mills theory to the conformally flat gravity described by the field $\chi$ [11]. This way the scale symmetry of Yang-Mills theory is restored at the cost of introducing a new field. At very short distances $\alpha_{s} \ll 1$ and the scale anomaly vanishes in usual perturbative gluodynamics. Effectively this means considering Yang-Mills theory in the flat space. At very long distances the theory resides in its physical vacuum $\chi=0$, see (17), which means that the spacetime is flat again. In between those extreme cases we can think of Yang-Mills field as a classical field propagating on a curved background. Indeed it has been found Ref. 23] that the coupling of the Yang-Mills theory on a curved background freezes at long distances.

The physical picture which has emerged from our study thus corresponds to color fields dynamically confined within a cavity by the interaction with self-coupled scalar glueball fields. This interaction regularizes the theory in the infrared region, and leads to the freezing of strong coupling at large distances.

It will be very interesting to study the properties of bound states in this "conformal bag model". While we checked that the model does have the corresponding solutions, so far we have not succeeded in finding them analytically.

A crucial test of the ideas presented in this paper can be performed on the lattice. Since $r_{0} \sim 1 / M_{0}$ corresponds to the size of the scalar glueball, and $M_{0}$ decreases as a function of $N_{c}$, we predict that the scalar glueball in $S U(4)$ gauge theory will have a larger size than in $S U(3)$. Unlike in $S U(3)$ theory, where the scalar glueball was found to have the smallest size (see e.g. 20]), in $S U(4)$ we expect all glueballs to have similar sizes. In contrast, in $S U(2)$ theory the size of the scalar glueball should become even smaller than in $S U(3)$. These predictions can be tested directly by measuring the glueball formfactors (three-point correlation functions), or indirectly by measuring the two-point correlation functions of the scalar gluon operators and by checking at what distances they approach the perturbative behavior. If the lattice results in gluodynamics confirm the validity of the effective theory advocated in this paper, it will be worthwhile to include the light quarks by putting the classical QCD Lagrangian on the curved background. This could then substitute a consistent theoretical approach to the study of infrared behavior in QCD. 


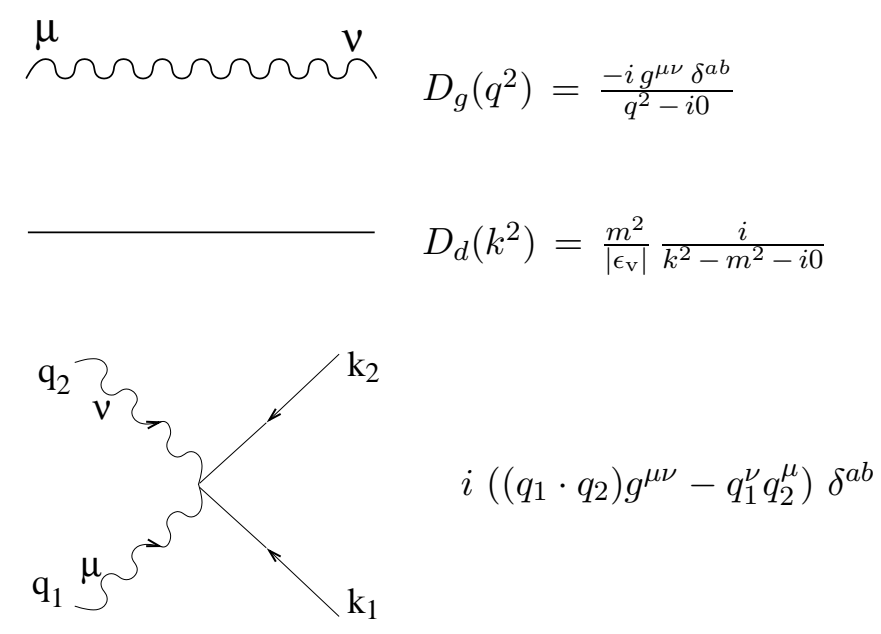

FIG. 4: Feynman rules for the dilaton effective theory.

\section{Acknowledgments}

We acknowledge interesting discussions on the subject with A. Gotsman, Yu. Kovchegov, U. Maor, M. Praszalowicz and D.T. Son. We are indebted to T.D. Lee for valuable comments and suggestions, and to H. Meyer and P. Petreczky for a discussion of the lattice data on glueballs. The work of D.K. and K.T. was supported by the U.S. Department of Energy under Contract No. DE-AC02-98CH10886. This research was supported in part by the GIF grant \# I-620-22.14/1999 and by Israeli Science Foundation, founded by the Israeli Academy of Science and Humanity. At the early stage the work of K.T. was sponsored by the U.S. Department of Energy under Grant No. DE-FG03-00ER41132.

\section{APPENDIX A: FEYNMAN RULES FOR THE DILATON LAGRANGIAN}

In Appendix A we list the Feynman rules for the Lagrangian (22) up to the quadratic terms in $\chi$, see Fig. 4. Here $a$ and $b$ are the color indexes. We observe that dilaton graphs do not violate the color symmetry. This is seen of course directly from the Lagrangian (22). 


\section{APPENDIX B: HIGHER ORDER CORRECTIONS TO THE GLUON POLARIZATION TENSOR}

In Appendix B we argue that the higher order corrections to the gluon polarization tensor have no singularities at the end-point of the dilaton spectrum $q^{2}=4 m^{2}$. Let us consider the diagram Fig. 5 for example.

We have

$$
\begin{aligned}
i \Pi_{\mu \nu}^{b}(q)= & i \Pi_{\mu \nu}(q) \\
= & \left(\frac{m^{2}}{\left|\epsilon_{\mathrm{v}}\right|}\right)^{2} \frac{1}{2 !} \int \frac{d^{4} p}{(2 \pi)^{4}} \frac{d^{4} k_{1}}{(2 \pi)^{4}} \frac{d^{4} k_{2}}{(2 \pi)^{4}} i\left(p^{\mu} q^{\rho}-(q p) g^{\mu \rho}\right) \frac{-i g^{\rho \lambda}}{p^{2}} \\
& \times i\left(p^{\nu} q^{\lambda}-(q p) g^{\nu \lambda}\right) \frac{i}{k_{1}^{2}-m^{2}} \frac{i}{k_{2}^{2}-m^{2}}(2 \pi)^{4} \delta\left(k_{1}+k_{2}+p-q\right) .
\end{aligned}
$$

Contracting Lorentz indexes and averaging over directions of $p$ it can be shown that $\Pi_{\mu \nu}(q)$ has the same transverse structure as displayed in (46). Making contractions in the definition (46) we arrive at

$$
\begin{aligned}
\operatorname{Im} \Pi(q)= & \frac{1}{3 q^{2}} \operatorname{Im} \Pi_{\mu}^{\mu}(q)=\frac{1}{3 q^{2}}\left(\frac{m^{2}}{\left|\epsilon_{\mathrm{v}}\right|}\right)^{2} \frac{1}{16 \pi} \int d^{4} k \int \frac{d^{4} p}{(2 \pi)^{3}} \\
& \times \delta(k+p-q) \delta\left(p^{2}\right) 2(p q)^{2} \sqrt{1-\frac{4 m^{2}}{M^{2}}} \\
= & \frac{1}{3 q^{2}}\left(\frac{m^{2}}{\left|\epsilon_{\mathrm{v}}\right|}\right)^{2} \frac{1}{64 \pi^{3}} \int d M^{2}|\vec{k}| \omega_{p}^{2} M_{q} \sqrt{1-\frac{4 m^{2}}{M^{2}}}
\end{aligned}
$$

where $M_{q}^{2}$ is the external gluon virtuality. Denote $t=M_{q}^{2}$. It is easily seen that

$$
\omega_{p}=|\vec{p}|=|\vec{k}|=\frac{t-M^{2}}{2 \sqrt{t}} .
$$

Integral in (B2) over $M^{2}$ in the range $4 m^{2} \leq M^{2} \leq t$ can be easily done giving somewhat lengthy result. Near the end-point of the spectrum the result of integration is

$$
\operatorname{Im} \Pi(t) \approx\left(\frac{m^{2}}{\left|\epsilon_{\mathrm{v}}\right|}\right)^{2} \frac{\left(t-4 m^{2}\right)^{9 / 2}}{140(6 \pi)^{3} m t^{2}} .
$$

The polarization tensor can be calculated using dispersion relation

$$
\Pi(q)=\left(q^{2}-4 m^{2}\right)^{5} \frac{1}{\pi} \int_{4 m^{2}}^{M_{0}^{2}} \frac{\operatorname{Im} \Pi(q)}{\left(t-q^{2}-i 0\right)\left(t-4 m^{2}\right)^{5}} d t .
$$

Dispersion relation can be applied only to a function which vanishes sufficiently fast at infinite radius in the complex plain of $t$. Therefore we apply it to a function $\operatorname{Im} \Pi(t) /\left(t-4 m^{2}\right)^{5}$ 


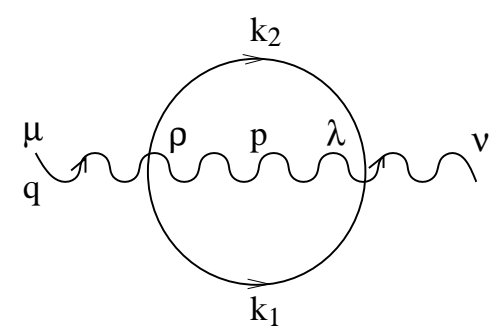

FIG. 5: Next-to-leading order diagram contributing to the gluon polarization tensor.

instead of $\operatorname{Im} \Pi(t)$. This procedure corresponds to the subtractions

$$
\Pi\left(q^{2}\right) \rightarrow \Pi\left(q^{2}\right)-\sum_{l=0}^{4} \frac{1}{l !} \Pi^{(l)}\left(4 m^{2}\right)\left(q^{2}-4 m^{2}\right)^{l} .
$$

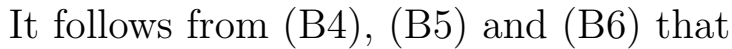

$$
\Pi(q) \propto\left(t-4 m^{2}\right)^{9 / 2} \rightarrow 0, \quad \text { as } t \rightarrow 4 m^{2}, \quad l \neq 0
$$

The term with $l=0$ is just the largest subtraction constant (cp. (43)).

Therefore we can safely expand (B2) in powers of $\lambda$. Integrating over $M^{2}$ and using dispersion relation (B5) with $m=0$ we obtain

$$
\Pi\left(Q^{2}\right)=\frac{Q^{4} m^{4}}{\left|\epsilon_{\mathrm{v}}\right|^{2}} \ln \frac{M_{0}^{2}+Q^{2}}{Q^{2}} \frac{1}{24(4 \pi)^{4}}+\text { const. }
$$

At $Q^{2}=M_{0}^{2}$ this contribution reaches its maximal value $\sim \lambda^{2}$ and thus parametrically and numerically suppressed with respect to the leading result (48).

We can easily extend our argument to higher order diagrams. Indeed, the introduction of additional dilaton lines can only bring in a factors of $M^{2} / m^{2}$ as can be seen from the gluon-dilaton vertex in the Appendix A and Fig. 1(b) of Ref. [16] for dilaton self interactions.

[1] D. J. Gross and F. Wilczek, Phys. Rev. Lett. 30 (1973) 1343; H. D. Politzer, Phys. Rev. Lett. 30, 1346 (1973).

[2] I.A. Batalin, S.G. Matinyan and G. Savvidy, Sov. J. Nucl. Phys. 26 (1977) 214; S.G. Matinyan and G.K. Savvidy, Nucl. Phys. B134 (1978) 539; G.K. Savvidy Phys. Lett. B71 (1977) 133.

[3] N. K. Nielsen and P. Olesen, Nucl. Phys. B 144, 376 (1978). 
[4] W. Y. Tsai, Phys. Rev. D 7, 1945 (1973).

[5] V. N. Gribov, Nucl. Phys. B 139, 1 (1978).

[6] A. Chodos, R. L. Jaffe, K. Johnson, C. B. Thorn and V. F. Weisskopf, Phys. Rev. D 9, 3471 (1974).

[7] N. D. Birrell and P. C. W. Davies, "Quantum Fields In Curved Space," Cambridge, UK: Univ. Press (1982).

[8] R. Friedberg and T. D. Lee, Phys. Rev. D 15, 1694 (1977); R. Friedberg and T. D. Lee, Phys. Rev. D 16, 1096 (1977).

[9] T. D. Lee and G. C. Wick, Phys. Rev. D 9, 2291 (1974).

[10] H. Pagels and E. Tomboulis, Nucl. Phys. B 143, 485 (1978).

[11] A.A. Migdal and M.A. Shifman, Phys. Lett. B114 (1982) 445.

[12] J. Schechter, Phys. Rev. D 21, 3393 (1980);

A. Salomone, J. Schechter and T. Tudron, Phys. Rev. D 23, 1143 (1981);

H. Gomm, P. Jain, R. Johnson and J. Schechter, Phys. Rev. D 33, 801 (1986).

[13] M.A. Shifman, A.I. Vainshtein, V.I. Zakharov, JETP Lett. 27 (1978) 55, Nucl. Phys. B147 (1979) 385, Nucl. Phys. B147 (1979) 448, Nucl. Phys. B147 (1979) 519; M.A. Shifman, A.I. Vainshtein, M.B. Voloshin, V.I. Zakharov, Phys. Lett. B77 (1978) 80.

[14] V.A. Novikov, M.A. Shifman, A.I. Vainshtein, V.I. Zakharov, Phys. Lett. B86 (1979) 347, Nucl. Phys. B165 (1980) 67, Nucl. Phys. B191 (1981) 301.

[15] J. Ellis, Nucl. Phys. B22 (1970) 478; R.J. Crewther, Phys. Lett. B33 (1970) 305, Phys. Rev. Lett. 28 (1972) 1421; M.S. Chanowitz and J. Ellis, Phys. Lett. B40 (1972) 397; Phys. Rev. D7 (1973) 2490; C. Callan, S. Coleman and R. Jackiw, Ann. Phys. 59 (1970) 42; S. Coleman and R. Jackiw, Ann. Phys. 67 (1971) 552.

[16] D. Kharzeev, E. Levin and K. Tuchin, Phys. Lett. B 547, 21 (2002) arXiv:hep-ph/0204274.

[17] H. Fujii and D. Kharzeev, Phys. Rev. D 60 (1999) 114039

[18] J. Ellis, H. Fujii and D. Kharzeev, arXiv:hep-ph/9909322.

[19] S. J. Brodsky, S. Menke, C. Merino and J. Rathsman, Phys. Rev. D 67, 055008 (2003) arXiv:hep-ph/0212078.

[20] M. J. Teper, arXiv:hep-th/9812187; Y. Chen et al., arXiv:hep-lat/0310013.

[21] Yu.L. Dokshitzer, hep-ph/9812252

[22] E. V. Shuryak, Phys. Lett. B 486, 378 (2000) arXiv:hep-ph/0001189. 
[23] E. Elizalde, S. D. Odintsov and A. Romeo, Phys. Rev. D 54, 4152 (1996) arXiv:hep-th/9607189.

[24] M. Peskin and D. Schroeder, "An Introduction to Quantum Field Theory", Perseus Books.

[25] L.D. Landau and E.M. Lifshitz, "Classical theory of fields". 